\title{
Meta classifications for Acute Inflammations Data Set
}

\author{
Dr.G.Ayyappan \\ Assistant Professor, Department of Information Technology, \\ Bharath University \\ K.SivaKumar \\ SIPS Technologies, \\ Chennai, Tamil Nadu, India.
}

\begin{abstract}
This research work presents a decision making of healthcare operational system by using machine learning classifiers algorithm to predict the decision making in comparison to the actual decision making. This model may help to doctor for making the best decisions. This model helps us to predict Acute Inflammations. The results show that Bagging, logitBoost and Multiclassclassifier for this case study generates highest accuracy of $48.75 \%$.
\end{abstract}

\section{INTRODUCTION}

Machine learning in today's healthcare is unavoidable. Today's healthcare needs effective methods and research methodologies to save lives, reduce the cost of the healthcare services and early discoveries of contagious diseases. Now a day's instances in healthcare such as medical image processing and analyzing, predicting healthcare operational decisions, dosage trials for intravenous tumor treatment detection and management of prostate cancer. In this research work applied in weka 3.8.3 version for Meta classification method by applying various kernels namely Polykernel, Normalized Polykernel, Puk, and RBF Kernel were applied to calculate for predicting caesarian section operational decisions.

In this paper organizes section one has related works and brief introduction of this fields, section two presents Materials and Methods, the section three describes results and discussions and the section four presents conclusion.

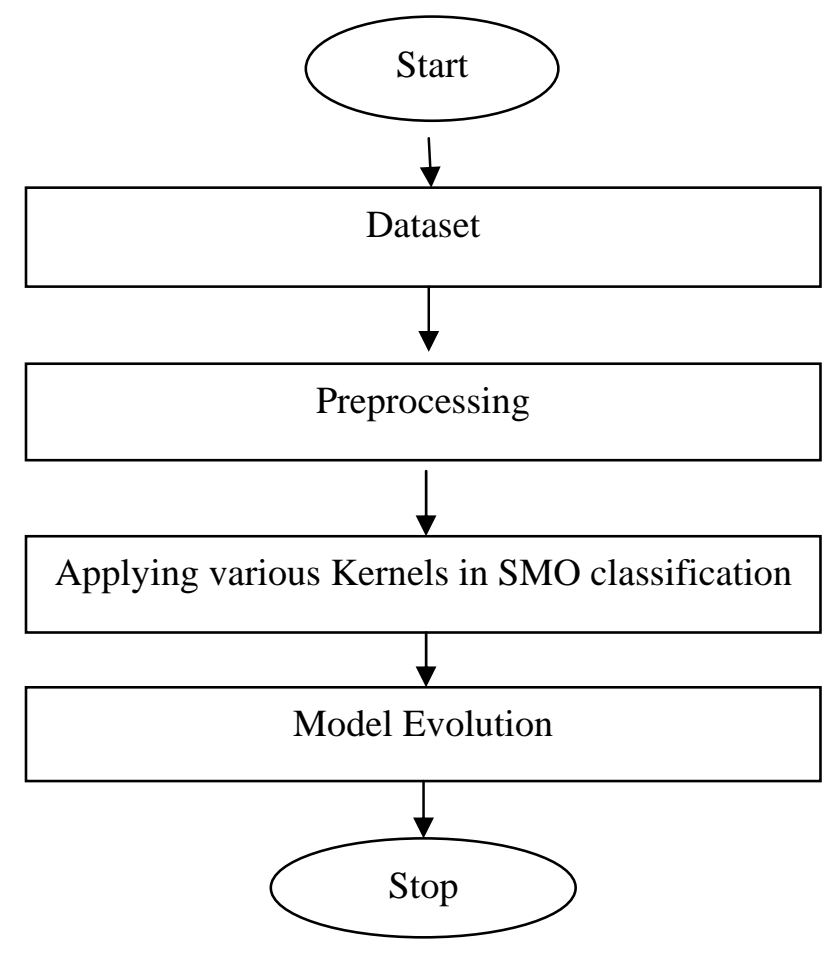

Figure 1 Architecture of Proposed method 


\section{RESULTS AND DISCUSSIONS}

In this work various machine learning meta algorithms namely AdaboostM1, Bagging, RandomCommitte, logitBoost and Multiclassclassifierwere used to calculate for predicting caesarian section operational decisions.

Table 2 Accuracy levels of Caesarian Section Classification Dataset Data Set

\begin{tabular}{|c|c|c|}
\hline S.No & Mata Classification & Accuracy level \\
\hline 1 & AdaboostM1 & $42 \%$ \\
\hline 2 & Bagging & $48.75 \%$ \\
\hline 3 & RandomCommitte & $38.75 \%$ \\
\hline 4 & logitBoost & $48.75 \%$ \\
\hline 5 & Multiclassclassifier & $48.75 \%$ \\
\hline
\end{tabular}

In this work various machine learning algorithms namely AdaboostM1 has $42 \%$,Bagging has $48.75 \%$, RandomCommitte has $38.75 \%$,logitBoost has $48.75 \%$ and Multiclassclassifier has $48.75 \%$ were calculated for predicting caesarian section operational decisions.

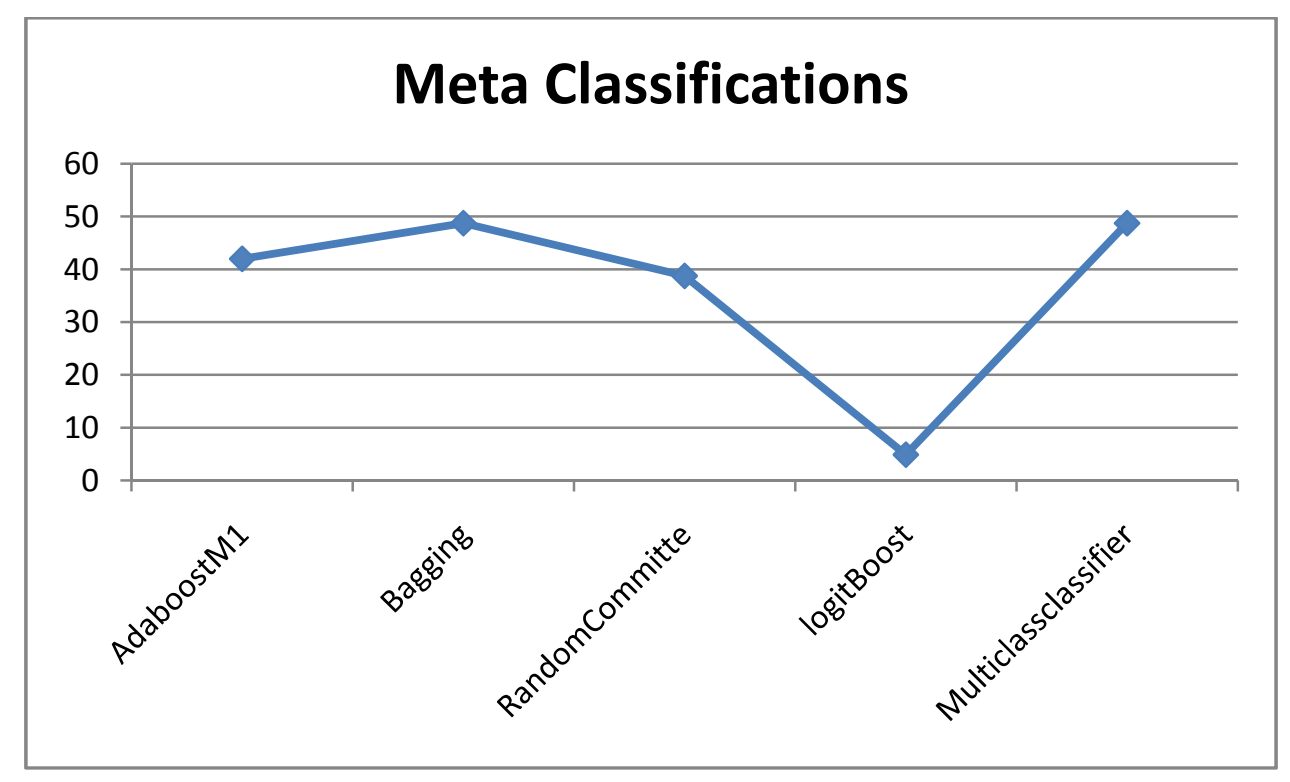

Figure 2 Accuracy levels of various classifications

\section{CONCLUSION}

This paper clearly shows that Bagging, logitBoost and multiclassclassifier achieved the best accuracy rates by predicting 48.75 cases correctly compare than other models. So, this research work recommended for decision making based on the Bagging, logitBoost and multiclassclassifier classifications.

\section{REFERENCES}

[1] (PDF) Performance Evaluation of Supervised Machine Learning Classifiers for Predicting Healthcare Operational Decisions. Available

from: https://www.researchgate.net/publication/329515048_Performance_Evaluation_of_Supervised_Machine_Learning_Classifiers_for_Pr edicting_Healthcare_Operational_Decisions [accessed Jan 06 2019].

[2] Boris Milovic, MilanMilovic. Prediction and Decision Making in Health Care using Data Mining, International Journal of Public Health Science (IJPHS), Vol. 1, No. 2, December 2012, pp. 69 78

[3] Fayyad, U., Shapiro, G. P., \& Smyth, P. (1996). From Data Mining to Knowledge Discovery in Databases. American Association for Artificial Intelligence, 37-54.

[4] H. Jiawei and K. Micheline, Data Mining: Concepts and Techniques, vol. 2, Morgan Kaufmann Publishers, 2006.

[5] Candelieri, A., Dolce, G., Riganello, F., \&Sannita, W. G. (2011). Data Mining in Neurology. In KnowledgeOriented Applications in Data Mining (pp. 261-276). InTech.

[6] Brady E. Hamilton, Ph.D.; Joyce A. Martin, M.P.H.; and Stephanie J. Ventura, M.A., Division of Vital Statistics, Births: Preliminary Data for 2007, National Vital Statistics Report. [7] Births: Provisional Data for 2017 USA. CDC. May 2018. Retrieved 18 May 2018.

[7] World Health Organization (WHO) statement "Should there be a limit on Caesareans?". BBC News. 30 June 2010.

[8] Andrew Simm, Darly Mathew, Caesarian section: techniques and complications, Obstetrics, Gynaecology\& Reproductive Medicine, Volume 18, Issue 4, April 2008, Pages 93-98.

[9] FarhadSoleimanianGharehchopogh, PeymanMohammadi, ParvinHakimi, Application of Decision Tree Algorithm for Data Mining in Healthcare Operations: A Case Study, International Journal of Computer Applications (0975 - 8887) Volume 52 - No. 6, August 2012,Pages 21-26. 